\title{
GENETIC VARIATION OF Apis mellifera FROM SERBIA INFERRED FROM MITOCHONDRIAL ANALYSIS
}

\author{
Irene Muñoz ${ }^{1}$, Jevrosima Stevanovic ${ }^{2}$, \\ Zoran Stanimirovice ${ }^{2}$ Pilar De la Rúa ${ }^{1}$ \\ ${ }^{1}$ Dpto. de Zoología y Antropología Física, Facultad de Veterinaria, \\ Universidad de Murcia, 30100 Murcia, Spain. \\ ${ }^{2}$ Department of Biology, Faculty of Veterinary Medicine, \\ University of Belgrade, Bul. oslobodjenja 18, 11000 Belgrade, Serbia. \\ e-mail:pdelarua@um.es
}

Received 16 November 2011; accepted 21 March 2012

$\mathrm{S} \mathrm{u} \mathrm{m} \mathrm{m} \mathrm{a} \mathrm{r} \mathrm{y}$

Two honeybee subspecies inhabit Serbia; Apis mellifera carnica and A. m. macedonica. Both belong to eastern Mediterranean (C) evolutionary lineage. Furthermore three Serbian honeybee ecotypes restricted to particular regions, were defined through morphometry and cytogenetic analyses. In this study, mitochondrial data have been used to analyze the molecular diversity of the honeybee population from Serbia. Seven haplotypes of the $\mathrm{C}$ evolutionary lineage have been found, two of them are newly described ( $\mathrm{C} 2 \mathrm{o}$ and $\mathrm{C} 2 \mathrm{p})$ and restricted to two regions, which ultimately increased the number of haplotypes found in this lineage. Comparisons with surrounding honeybee populations suggest a hybrid situation between A. m. carnica and A. m. macedonica and also introgression from $A$. m. ligustica. The results should be considered when dealing with future conservation strategies, and for pathogen-parasite-tolerant breeding programs.

Keywords: Apis mellifera carnica, Apis mellifera macedonica, mitochondrial DNA, intergenic region, haplotypes, sequencing, population genetics.

\section{INTRODUCTION}

The honeybee Apis mellifera is endemic to Africa, Europe and Western Asia, but actually this species has spread worldwide due to multiple migrations and introductions (Moritz et al., 2005; Whitfield et al., 2006). Around 29 subspecies have been recognized based on morphological and ecological data (Engel, 1999; Sheppard and Meixner, 2003). These species have been grouped into four morphometric groups (Ruttner, 1988): the African group (A), the Northern and Western European group (M), the East Mediterranean group (C) and the Oriental group (O). The morphometric C-group included the subspecies of the Balkan region, plain of the Danube and diverse areas such as Sicily, the Apennine Peninsula and the Ukraine (Ruttner, 1988).
The morphometric C-group consisted of five honeybee subspecies: $A$. m. carnica, A. m. ligustica, A. m. macedonica, A. m. cecropia and A. m. siciliana (lately included in the African group based on molecular analyses, Sinacori et al., 1998). Different studies have recently been done to depict the relationships among these subspecies, using molecular grounds (Bouga et al., 2005; Ivanova et al., 2010; Martimianakis et al., 2011).

Serbia is situated in the middle of the distribution area of the C-group. It is located in Southeastern Europe, covering two distinct geographic areas: the Pannonian Plain in the north between the Sava and Danube rivers and a southern mountainous part covering the central part of the Balkan Peninsula. The climate in Serbia varies from the continental type 
in the north to moderate-continental in the south. In relation to beekeeping in the northern and central parts of Serbia, beekeepers practice extensive seasonal movements of their colonies. Besides intra-regional movements, they often migrate their colonies to Eastern Serbia. However, beekeepers in Eastern Serbia rarely move their colonies. When they do move them, the colonies are only moved short distances, whilst in Southern Serbia (including Syenichko-Peshterski Plateau) migratory beekeeping is not practiced.

Two subspecies of $A$. mellifera inhabit the territory of Serbia, A. m. carnica and A. $m$. macedonica, both belonging to the East Mediterranean $\mathrm{C}$ evolutionary lineage. Based on morphometric analyses (Ruttner, 1988), A. m. carnica is considered to be distributed across Central-eastern European countries such as Austria, Hungary, Romania, Bulgaria and former Yugoslavia (Slovenia, Croatia, Serbia, Bosnia and Herzegovina, Montenegro, the Republic of Macedonia), whilst $A$. m. macedonica was described as inhabiting parts of the Ukraine, Romania, Bulgaria, parts of former Yugoslavia and Northern Greece, giving no exact border between $A$. $m$. carnica and A. m. macedonica. Furthermore, cytogenetic and morpho - metric analyses (Stanimirovic et al., 1999a,b; Stevanovic, 2002; Stanimirovic et al., 2005a), as well as behavioural analyses (Pejovic, 2001; Cirkovic, 2002; Stanimirovic et al., 2002, 2005b; Stevanovic, 2007) described the presence of three honeybee ecotypes (defined in terms of locally adapted populations within a subspecies with a particular distribution): Banat (B), Timok (T) and Syenichko-Peshterski (S). Cytogenetic research revealed differences in the biometric and ultrastructural organization of chromosomes between the B and S honeybee ecotypes (Stanimirovic et al., 1999a,b). Chromosomal analyses comprising the three honeybee ecotypes from Serbia demonstrated the existence of a G-band polymorphism (Stanimirovic et al., 2005a). These observations were in accordance with previous results obtained by morphometric analysis of the same ecotypes using 30 morphometric characters (Stevanovic, 2002). This high morphometric variance suggests that the honeybee populations from Serbia may play a pivotal role in the understanding of the biogeographic transition of the subspecies carnica and macedonica.

Giving these preliminary results, the two aims of the present work were: 1) to test whether the Serbian honeybee ecotypes defined through cytogenetic and morphometric analyses are genetically differentiated, and 2) to depict the relationship of the honeybees from Serbia in relation to neighbouring $A$. mellifera subspecies.

\section{MATERIAL AND METHODS}

\section{Sampling and DNA extraction}

Adult honeybee workers were sampled from three Serbian regions where the ecotypes have been described: Banat (B), Timok (T) and Syenichko-Perhterski (S). Samples from the Southeastern (SE) region were also included (Tab. 1 and Fig. 1). A total of 37 colonies were sampled in Serbia and 24 reference honeybee colonies were additionally included from Italy ( $A$. m. ligustica, $\mathrm{N}=5$ ), Croatia (A. m. carnica, $\mathrm{N}=5)$, Bosnia and Herzegovina (A. m. carnica, $\mathrm{N}=5$ ), Albania (hybrid population of $A$. m. carnica and A. $m$. macedonica, $\mathrm{N}=4$, Dedej et al., 2000) and the Republic of Macedonia (A. m. macedonica, $\mathrm{N}=5$ ) for comparison purposes (Tab. 1). Worker honeybee samples were preserved in absolute ethanol and kept at $-20^{\circ} \mathrm{C}$. Total DNA was extracted from three right legs of each honeybee worker using a 5\% Chelex solution (Walsh et al., 1991).

\section{Mitochondrial DNA analysis}

A single honeybee worker per colony was used for mtDNA analysis. The tRNA $^{\text {leu }}-\operatorname{cox} 2$ intergenic region were PCR amplified with the primers E2 (5'-GGCAGAATAAGTGCATTG -3') located at the 5 ' end of the gene tRNA ${ }^{\text {leu }}$ and 
Collection sites and mtDNA haplotype found in the sampled colonies of $A$. mellifera

\begin{tabular}{|c|c|c|c|c|}
\hline Sample & Locality & Region/Country & Geographical Coord. & mtDNA \\
\hline SER01 & Belosavci & Banat/Serbia & $44^{\circ} 19^{\prime} \mathrm{N} 20^{\circ} 41^{\prime} \mathrm{E}$ & $\mathrm{C} 2 \mathrm{e}$ \\
\hline SER02 & Dumbovo & Banat/Serbia & $45^{\circ} 34^{\prime} \mathrm{N} \quad 19^{\circ} 39^{\prime} \mathrm{E}$ & C2e \\
\hline SERO3 & Curug & Banat/Serbia & $45^{\circ} 12^{\prime} N \quad 19^{\circ} 43^{\prime} \mathrm{E}$ & C2d \\
\hline SER04 & Dublje & Banat/Serbia & $44^{\circ} 47^{\prime} \mathrm{N} \quad 19^{\circ} 31^{\prime} \mathrm{E}$ & C2d \\
\hline SER05 & Ripanj & Banat/Serbia & $44^{\circ} 38^{\prime} \mathrm{N} 20^{\circ} 32^{\prime} \mathrm{E}$ & C2d \\
\hline SER06 & Cerak & Banat/Serbia & $44^{\circ} 44^{\prime} \mathrm{N} 20^{\circ} 24^{\prime} \mathrm{E}$ & C2i \\
\hline SER07 & Sevarice & Banat/Serbia & $44^{\circ} 52^{\prime} \mathrm{N} \quad 19^{\circ} 39^{\prime} \mathrm{E}$ & $\mathrm{C} 2 \mathrm{O}$ \\
\hline SER08 & Veliko Gradiste & Banat/Serbia & 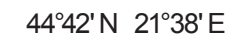 & C2d \\
\hline SER10 & Sedlari & Banat/Serbia & $44^{\circ} 18^{\prime} N \quad 19^{\circ} 53^{\prime} \mathrm{E}$ & C2d \\
\hline SER11 & Valjevo & Banat/Serbia & $44^{\circ} 16^{\prime} \mathrm{N} \quad 19^{\circ} 53^{\prime} \mathrm{E}$ & C2d \\
\hline SER19 & Sabac & Banat/Serbia & $44^{\circ} 52^{\prime} \mathrm{N} \quad 19^{\circ} 42^{\prime} \mathrm{E}$ & $\mathrm{C} 2 \mathrm{e}$ \\
\hline SER20 & Vrsac & Banat/Serbia & $45^{\circ} 07^{\prime} \mathrm{N} 21^{\circ} 18^{\prime} \mathrm{E}$ & C2d \\
\hline SER21 & Pancevo & Banat/Serbia & $44^{\circ} 51^{\prime} \mathrm{N} 20^{\circ} 39^{\prime} \mathrm{E}$ & C2d \\
\hline SERO9 & Pester & Syenichko-Peshterski/Serbia & $43^{\circ} 02^{\prime} \mathrm{N} 20^{\circ} 23^{\prime} \mathrm{E}$ & C2d \\
\hline SER32 & Kocarnik & Syenichko-Peshterski/Serbia & $42^{\circ} 59^{\prime} \mathrm{N} 20^{\circ} 19^{\prime} \mathrm{E}$ & C2d \\
\hline SER33 & Borostica & Syenichko-Peshterski/Serbia & $43^{\circ} 02^{\prime} \mathrm{N} 20^{\circ} 06^{\prime} \mathrm{E}$ & $\mathrm{C} 2 \mathrm{e}$ \\
\hline SER34 & Dubovo & Syenichko-Peshterski/Serbia & 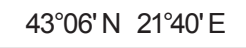 & C2d \\
\hline SER35 & Cvijetlje & Syenichko-Peshterski/Serbia & $43^{\circ} 08^{\prime} \mathrm{N} 20^{\circ} 31^{\prime} \mathrm{E}$ & $\mathrm{C} 2 \mathrm{e}$ \\
\hline SER36 & Livadak & Syenichko-Peshterski/Serbia & $42^{\circ} 59^{\prime} \mathrm{N} 20^{\circ} 19^{\prime} \mathrm{E}$ & C2e \\
\hline SER37 & Grabovica & Syenichko-Peshterski/Serbia & $42^{\circ} 18^{\prime} N \quad 18^{\circ} 53^{\prime} \mathrm{E}$ & $\mathrm{C} 2 \mathrm{e}$ \\
\hline SER38 & Sare & Syenichko-Peshterski/Serbia & $43^{\circ} 16^{\prime} \mathrm{N} 20^{\circ} 13^{\prime} \mathrm{E}$ & C2d \\
\hline SER39 & Pavlje & Syenichko-Peshterski/Serbia & $43^{\circ} 17^{\prime} N 20^{\circ} 36^{\prime} \mathrm{E}$ & C2p \\
\hline SER40 & Gonje & Syenichko-Peshterski/Serbia & $43^{\circ} 17^{\prime} \mathrm{N} \quad 19^{\circ} 54^{\prime} \mathrm{E}$ & C2p \\
\hline SER12 & llino & Timok/Serbia & 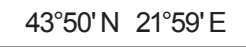 & C2d \\
\hline SER13 & Krivi Vir & Timok/Serbia & $43^{\circ} 49^{\prime} \mathrm{N} 21^{\circ} 44^{\prime} \mathrm{E}$ & Cla \\
\hline SER14 & Lukovo & Timok/Serbia & $43^{\circ} 48^{\prime} N 21^{\circ} 49^{\prime} E$ & $\mathrm{C} 2 \mathrm{c}$ \\
\hline SER15 & Stupanj & Timok/Serbia & 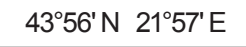 & C2d \\
\hline SER16 & Metris & Timok/Serbia & $43^{\circ} 54^{\prime} \mathrm{N} 22^{\circ} 11^{\prime} \mathrm{E}$ & $\mathrm{C} 2 \mathrm{i}$ \\
\hline SER17 & Lubnica & Timok/Serbia & 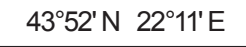 & C2d \\
\hline SER22 & Vladicin Han & Southeast/Serbia & $42^{\circ} 42^{\prime} \mathrm{N} 22^{\circ} 03^{\prime} \mathrm{E}$ & C2d \\
\hline SER23 & Presevo & Southeast/Serbia & $42^{\circ} 18^{\prime} \mathrm{N} 21^{\circ} 39^{\prime} \mathrm{E}$ & C2d \\
\hline SER24 & Pirot & Southeast/Serbia & $43^{\circ} 09^{\prime} \mathrm{N} 22^{\circ} 36^{\prime} \mathrm{E}$ & C2d \\
\hline SER26 & Vranjska banja & Southeast/Serbia & $42^{\circ} 33^{\prime} \mathrm{N} 22^{\circ} 01^{\prime} \mathrm{E}$ & $\mathrm{C} 2 \mathrm{~d}$ \\
\hline
\end{tabular}


Table 1. Continued

\begin{tabular}{|c|c|c|c|c|}
\hline SER27 & Leskovac & Southeast/Serbia & $42^{\circ} 59^{\prime} \mathrm{N} 21^{\circ} 57^{\prime} \mathrm{E}$ & $\mathrm{C} 2 \mathrm{~d}$ \\
\hline SER28 & Nis & Southeast/Serbia & $43^{\circ} 19^{\prime} \mathrm{N} 21^{\circ} 53^{\prime} \mathrm{E}$ & $\mathrm{C} 2 \mathrm{e}$ \\
\hline SER30 & Doljevac & Southeast/Serbia & $43^{\circ} 12^{\prime} N 21^{\circ} 48^{\prime} \mathrm{E}$ & $\mathrm{C} 2 \mathrm{~d}$ \\
\hline SER31 & Sinkovce & Southeast/Serbia & $42^{\circ} 55^{\prime} \mathrm{N} 21^{\circ} 43^{\prime} \mathrm{E}$ & $\mathrm{C} 2 \mathrm{e}$ \\
\hline ARU03 & Perugia & Italy & $43^{\circ} 06^{\prime} \mathrm{N} \quad 12^{\circ} 23^{\prime} \mathrm{E}$ & $\mathrm{Cla}$ \\
\hline CAM01 & Camugnano & Italy & $44^{\circ} 10^{\prime} \mathrm{N} \quad 11^{\circ} 05^{\prime} \mathrm{E}$ & $\mathrm{Cla}$ \\
\hline CAM02 & Camugnano & Italy & $44^{\circ} 10^{\prime} \mathrm{N} \quad 11^{\circ} 05^{\prime} \mathrm{E}$ & $\mathrm{Cla}$ \\
\hline CAM03 & Camugnano & Italy & $44^{\circ} 10^{\prime} \mathrm{N} \quad 11^{\circ} 05^{\prime} \mathrm{E}$ & $\mathrm{Cla}$ \\
\hline CAM04 & Camugnano & Italy & $44^{\circ} 10^{\prime} \mathrm{N} 11^{\circ} 05^{\prime} \mathrm{E}$ & C1a \\
\hline BRA04 & Brac & Croatia & $43^{\circ} 19^{\prime} \mathrm{N} \quad 16^{\circ} 46^{\prime} \mathrm{E}$ & $\mathrm{C} 2 \mathrm{c}$ \\
\hline KRC02 & Korcula & Croatia & $42^{\circ} 56^{\prime} \mathrm{N} \quad 17^{\circ} 07^{\prime} \mathrm{E}$ & $\mathrm{C} 2 \mathrm{e}$ \\
\hline KRC03 & Korcula & Croatia & $42^{\circ} 56^{\prime} \mathrm{N} \quad 17^{\circ} 07^{\prime} \mathrm{E}$ & $\mathrm{C} 2 \mathrm{e}$ \\
\hline MLS04 & Mali Losini & Croatia & $44^{\circ} 31^{\prime} \mathrm{N} \quad 14^{\circ} 27^{\prime} \mathrm{E}$ & $\mathrm{C} 2 \mathrm{e}$ \\
\hline STV04 & Sutivanac & Croatia & $45^{\circ} 06^{\prime} \mathrm{N} 13^{\circ} 57^{\prime} \mathrm{E}$ & $\mathrm{Cla}$ \\
\hline BOS01 & llova & Bosnia and Herzegovina & $44^{\circ} 45^{\prime} \mathrm{N} \quad 17^{\circ} 37^{\prime} \mathrm{E}$ & $\mathrm{C} 2 \mathrm{~d}$ \\
\hline BOSO2 & Usce & Bosnia and Herzegovina & $43^{\circ} 50^{\prime} \mathrm{N} \quad 19^{\circ} 18^{\prime} \mathrm{E}$ & $\mathrm{C} 2 \mathrm{~d}$ \\
\hline BOS03 & Arandelovo & Bosnia and Herzegovina & $44^{\circ} 53^{\prime} N \quad 17^{\circ} 30^{\prime} E$ & $\mathrm{C} 2 \mathrm{~d}$ \\
\hline BOS04 & Trebinje & Bosnia and Herzegovina & $42^{\circ} 42^{\prime} N \quad 18^{\circ} 20^{\prime} \mathrm{E}$ & $\mathrm{C} 2 \mathrm{~d}$ \\
\hline BOS05 & Gacko & Bosnia and Herzegovina & $43^{\circ} 07^{\prime} N \quad 18^{\circ} 29^{\prime} \mathrm{E}$ & $\mathrm{C} 2 \mathrm{~d}$ \\
\hline SAR01 & Sarande & Albania & $39^{\circ} 52^{\prime} \mathrm{N} 20^{\circ} 00^{\prime} \mathrm{E}$ & $\mathrm{C} 2 \mathrm{~d}$ \\
\hline SAR02 & Sarande & Albania & $39^{\circ} 52^{\prime} \mathrm{N} \quad 2^{\circ} 00^{\prime} \mathrm{E}$ & $\mathrm{C} 2 \mathrm{~d}$ \\
\hline SAR03 & Sarande & Albania & $39^{\circ} 52^{\prime} \mathrm{N} \quad 2^{\circ} 00^{\prime} \mathrm{E}$ & $\mathrm{C} 2 \mathrm{i}$ \\
\hline SAR04 & Sarande & Albania & $39^{\circ} 52^{\prime} \mathrm{N} \quad 2^{\circ} 00^{\prime} \mathrm{E}$ & $\mathrm{C} 2 \mathrm{i}$ \\
\hline MAC02 & Probistip & Macedonia & $41^{\circ} 57^{\prime} \mathrm{N} 22^{\circ} 10^{\prime} \mathrm{E}$ & C2d \\
\hline MAC03 & Kriva Palanka & Macedonia & $42^{\circ} 10^{\prime} \mathrm{N} 22^{\circ} 19^{\prime} \mathrm{E}$ & $\mathrm{C} 2 \mathrm{~d}$ \\
\hline MACO4 & Strumica & Macedonia & $41^{\circ} 24^{\prime} \mathrm{N} 22^{\circ} 37^{\prime} \mathrm{E}$ & $\mathrm{C} 2 \mathrm{~d}$ \\
\hline MAC05 & Demir Hisar & Macedonia & $41^{\circ} 11^{\prime} \mathrm{N} 21^{\circ} 12^{\prime} \mathrm{E}$ & $\mathrm{C} 2 \mathrm{~d}$ \\
\hline MAC09 & Kumanovo & Macedonia & $42^{\circ} 06^{\prime} \mathrm{N} 21^{\circ} 42^{\prime} \mathrm{E}$ & $\mathrm{C} 2 \mathrm{~d}$ \\
\hline
\end{tabular}

H2 (5'-CAATATCATTGATGACC-3') located close to the 5 ' end of the gene cox 2 (Garnery et al., 1991) in a total volume of $12.5 \mu \mathrm{L}$, containing $2 \mu \mathrm{L}$ DNA, $10 \mathrm{mM}$ Tris- $\mathrm{HCl}, 50 \mathrm{mM} \mathrm{KCl}, 1.5 \mathrm{mM} \mathrm{MgCl} 2,0.2$ $\mathrm{mM}$ of each dNTP, $0.5 \mu \mathrm{M}$ of each primer and $1.25 \mathrm{U}$ of puReTaq DNA polymerase (GE Healthcare). The program cycle was as follows: denaturation for $5 \mathrm{~min}$ at $95^{\circ} \mathrm{C}$, 36 cycles of $45 \mathrm{~s}$ at $95^{\circ} \mathrm{C}, 60 \mathrm{~s}$ annealing at $47^{\circ} \mathrm{C}$, and extension for $1 \min 30 \mathrm{~s}$ at $72^{\circ} \mathrm{C}$ followed by a final elongation step of $10 \mathrm{~min}$ at $72^{\circ} \mathrm{C}$. Amplicons of each sample were purified with isopropanol and ammonium acetate and submitted to sequencing (Secugen S.L., Madrid, Spain).

Multiple alignments were done by using the online version of the multiple alignment program for amino acid or nucleotide sequences (MAFFT version 6, 


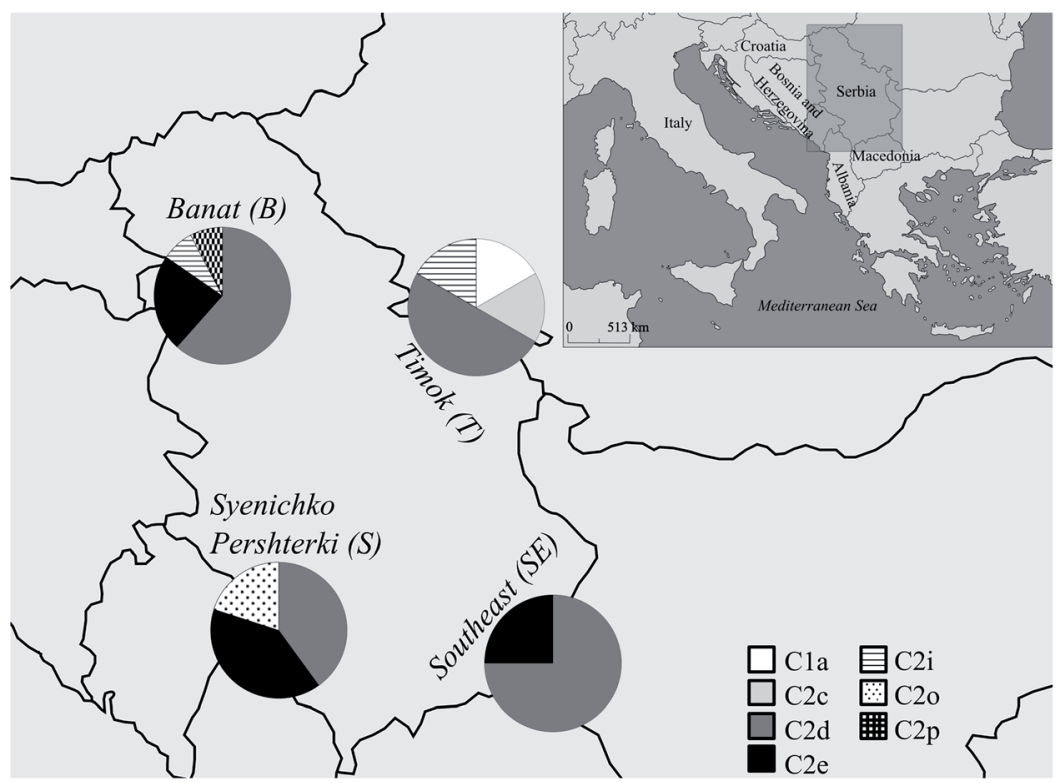

Fig. 1. Haplotype frequency pie charts in the different sampled regions from Serbia: Banat $(\mathrm{N}=13)$, Timok $(\mathrm{N}=6)$, Syenichko-Persterki $(\mathrm{N}=10)$ and Southeast $(\mathrm{N}=8)$.

KaToh and Toh, 2008) including published sequences for comparison (Franck et al., 2000; Sušnik et al., 2004; Muñoz et al., 2009). Haplotype networks were constructed using the median joining algorithm (Bandelt et al., 1999) with the software NETWORK 4.1.0.7 (fluxusengineering.com).

\section{RESULTS}

The identified haplotypes with sequence data are showed in Table 1. All samples bear only one $\mathrm{Q}$ sequence in the analyzed mtDNA fragment corresponding to the predicted composition of this region. Seven polymorphic sites were detected (Tab. 2) within the sequenced 571-573 bp mtDNA fragment (sequences are deposited in the GeneBank database with accession numbers JQ977699-JQ977705, see Fig. 2). These seven polymorphic sites defined the seven haplotypes found in Serbia, two of them newly described and named $\mathrm{C} 2 \mathrm{o}$ and $\mathrm{C} 2 \mathrm{p}$ (after Franck et al., 2000). The new haplotypes share with the previously described C2e haplotype (Muñoz et al., 2009), a single nucleotide deletion in position $3475 \mathrm{bp}$ (Crozier and Crozier, 1993), but were distinguishable from $\mathrm{C} 2 \mathrm{e}$ for a $\mathrm{T} \rightarrow \mathrm{A}$ transversion at the position $3515 \mathrm{bp}$, and a $\mathrm{T} \rightarrow \mathrm{C}$ transition at the position $3450 \mathrm{bp}$ present in $\mathrm{C} 2 \mathrm{o}$ and C2p haplotypes, respectively (Tab. 2).

The C2d haplotype was spread all over Serbia. This haplotype was most frequent in samples from the B (0.615), $\mathrm{T}(0.500)$ and SE (0.750) regions. C2e haplotype was recorded in S, SE and $\mathrm{B}$ region with a frequency of $0.400,0.250$ and 0.231 , respectively. Particular haplotypes were found in $\mathrm{T}(\mathrm{C} 1 \mathrm{a}$ and $\mathrm{C} 2 \mathrm{c}), \mathrm{B}$ and $\mathrm{T}(\mathrm{C} 2 \mathrm{i})$, $\mathrm{S}(\mathrm{C} 2 \mathrm{p})$ and $\mathrm{B}(\mathrm{C} 2 \mathrm{o})$ regions. Serbia haplotype diversity ranged from 0.375 (SE) to 0.667 (T) (Tab. 3).

In the reference populations, $\mathrm{C} 2 \mathrm{~d}$ was detected in $A$. m. carnica and A. m. macedonica samples from Bosnia and Herzegovina and the Republic of Macedonia, respectively, whereas $\mathrm{C} 1 \mathrm{a}$ was uniquely observed in $A$. m. ligustica from Italy. C1a was also observed in A. m. carnica from Croatia together with haplotypes $\mathrm{C} 2 \mathrm{c}$ and $\mathrm{C} 2 \mathrm{e}$. Two haplotypes, $\mathrm{C} 2 \mathrm{~d}$ and $\mathrm{C} 2 \mathrm{i}$ were present in 
11111111112222222223333333333444444444455555555556666666666777777777788888888889999999999 123456789012345678901234567890123456789012345678901234567890123456789012345678901234567890123456789 C1a GGCAGAATAAGTGCATTGAACTTAAGATTCAAATATAAAGTATTTTTAAACTTTTATTAAAATTTCCCCACTTAATTCATATTAATTTAAAAATAAATT C2C GGCAGAATAAGTGCATTGAACTTAAGATTCAAATATAAAGTATTTTTAAACTTTTATTAAAATTTCCC-ACTTAATTCATATTAATTTAAAAATAAATT C2d GGCAGAATAAGTGCATTGAACTTAAGATTCAAATATAAAGTATTTTTAAACTTTTATTAAAATTTCCC-ACTTAATTCATATTAATTTAAAAATAAATT C2e GGCAGAATAAGTGCATTGAACTTAAGATTCAAATATAAAGTATTTTTAAACTTTTATTAAAATTTCCC-ACTTAATTCATATTAATTTAAAAATAAATT C2i GGCAGAATAAGTGCATTGAACTTAAGATTCAAATATAAAGTATTTTTAAACTTTTATTAAAATTTCCC-ACTTAATTCATATTAATTTAAAAATAAATT C20 GGCAGAATAAGTGCATTGAACTTAAGATTCAAATATAAAGTATTTTTAAACTTTTATTAAAATTTCCC-ACTTAATTCATATTAATTTAAAAATAAATT C2P GGCAGAATAAGTGCATTGAACTTAAGATTCAAATATAAAGTATTTTTAAACTTTTATTAAAATTTCCC-ACTTAATTCATATTAATCTAAAAATAAATT

111111111111111111111111111111111111111111111111111111111111111111111111111111111111111111111111111 000000000011111111112222222222333333333344444444445555555555666666666677777777778888888888999999999 012345678901234567890123456789012345678901234567890123456789012345678901234567890123456789012345678 C1a AATAACAATTTTTAATAAAATAAATAATTAATTTTATTTTTATATTGAATTTTAAATTCAATCTTAAAGATTTAATCTTTTTATTAAAATTAATAAATT C2c AATAACAATTTTTAATAAAATAAATAATTAATTTTATTTTTATATTGAATTTTAAATTCAATCTTAAAGATTTAATCTTTTTATTAAAATTAATAAATT C2d AATAACAATTTTTAATAAAATAAATAATTAATTTTATTTTTATATTGAATTTTAAATTCAATCTTAAAGATTTAATCTTTTTATTAAAATTAATAAATT C2e AATAACAATTTT-AATAAAATAAATAATTAATTTTATTTTTATATTGAATTTTAAATTCAATCTTAAAGATTTAATCTTTTTATTAAAATTAATAAATT C2i AATAACAATTTTTAATAAAATAAATAATTAATTTTATTTTTATATTGAATTTTAAATTCAATCTTAAAGATTTAATCTTTTTATTAAAATTAATAAATT C20 AATAACAATTTT-AATAAAATAAATAATTAATTTTATTTTTATATTGAATTTAAAATTCAATCTTAAAGATTTAATCTTTTTATTAAAATTAATAAATT C2P AATAACAATTTT-AATAAAATAAATAATTAATTTTATTTTTATATTGAATTTTAAATTCAATCTTAAAGATTTAATCTTTTTATTAAAATTAATAAAT'

122222222222222222222222222222222222222222222222222222222222222222222222222222222222222222222222222 90000000000111111111122222222223333333333444444444555555555566666666667777777777888888888899999999 901234567890123456789012345678901234567890123456789012345678901234567890123456789012345678901234567 C1a AATATAAAATAAAACAAAATATAACAGAATATATTTATTAAAATTTAATTTATTAAAATTTCCACATGATTTATATTTATATTTCAAGAATCAAATTCA C2c AATATAAAATAAAACAAAATATAACAGAATATATTTATTAAAATTTAATTTATTAAAATTTCCACATGATTTATATTTATATTTCAAGAATCAAATTCA C2d AATATAAAATAAAACAAAATATAACAGAATATATTTATTAAAATTTAATTTATTAAAATTTCCACATGATTCATATTTATATTTCAAGAATCAAATTCA C2e AATATAAAATAAAACAAAATATAACAGAATATATTTATTAAAATTTAATTTATTAAAATTTCCACATGATTCATATTTATATTTCAAGAATCAAATTCA C2i AATATAAAATAAAACAAAATATAACAAAATATATTTATTAAAATTTAATTTATTAAAATTTCCACATGATTCATATTTATATTTCAAGAATCAAATTCA C20 AATATAAAATAAAACAAAATATAACAGAATATATTTATTAAAATTTAATTTATTAAAATTTCCACATGATTCATATTTATATTTCAAGAATCAAATTCA C2P AATATAAAATAAAACAAAATATAACAGAATATATTTATTAAAATTTAATTTATTAAAATTTCCACATGATTCATATTTATATTTCAAGAATCAAATTCA

223333333333333333333333333333333333333333333333333333333333333333333333333333333333333333333333333 9900000000001111111111222222222333333333344444444455555555556666666666777777777788888888889999999 890123456789012345678901234567890123456789012345678901234567890123456789012345678901234567890123456 Cla TATTATGCTGATAATTTAATTTCATTTCATAATATAGTTATAATAATTATTATTATAATTTCAACATTAACTGTATATATTATTTTAGATTTATTTATA C2c TATTATGCTGATAATTTAATTTCATTTCATAATATAGTTATAATAATTATTATTATAATTTCAACATTAACTGTATATATTATTTTAGATTTATTTATA C2d TATTATGCTGATAATTTAATTTCATTTCATAATATAGTTATAATAATTATTATTATAATTTCAACATTAACTGTATATATTATTTTAGATTTATTTATA C2e TATTATGCTGATAATTTAATTCATTTCATAATATAGTTATAATAATTATTATTATAATTTCAACATTAACTGTATATATTATTTTAGATTTATTTATA C2i TATTATGCTGATAATTTAATTCATTTCATAATATAGTTATAATAATTATTATTATAATTTCAACATTAACTGTATATATTATTTTAGATTTATTTATA C20 TATTATGCTGATAATTTAATTTCATTTCATAATATAGTTATAATAATTATTATTATAATTTCAACATTAACTGTATATATTATTTTAGATTTATTTATA C2p TATTATGCTGATAATTTAATTTCATTTCATAATATAGTTATAATAATTATTATTATAATTTCAACATTAACTGTATATATTATTTTAGATTTATTTATA

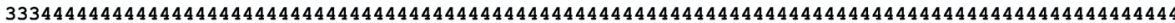
999000000000011111111112222222222333333333344444444445555555555666666666677777777778888888888999999 789012345678901234567890123456789012345678901234567890123456789012345678901234567890123456789012345 C1a AACAAATTCTCAAATTTATTTTTATTAAAAAATCATAATATTGAAATTATTTGAACAATTATTCCAATTATTATTCTATTAATTATTTGTTTTCCATCA C2c AACAAATTCTCAAATTTATTTTTATTAAAAAATCATAATATTGAAATTATTTGAACAATTATTCCAATTATTATTCTATTAATTATTTGTTTTCCATCA C2d AACAAATTTTCAAATTTATTTTTATTAAAAAATCATAATATTGAAATTATTTGAACAATTATTCCAATTATTATTCTATTAATTATTTGTTTTCCATCA C2e AACAAATTTTCAAATTTATTTTTATTAAAAAATCATAATATTGAAATTATTTGAACAATTATTCCAATTATTATTCTATTAATTATTTGTTTTCCATCA C2i AACAAATTTTCAAATTTATTTTTATTAAAAAATCATAATATTGAAATTATTTGAACAATTATTCCAATTATTATTCTATTAATTATTTGTTTTCCATCA C20 AACAAATTTTCAAATTTATTTTTATTAAAAAATCATAATATTGAAATTATTTGAACAATTATTCCAATTATTATTCTATTAATTATTTGTTTTCCATCA C2p AACAAATTTTCAAATTTATTTTTATTAAAAAATCATAATATTGAAATTATTTGAACAATTATTCCAATTATTATTCTATTAATTATTTGTTTTCCATCA

444455555555555555555555555555555555555555555555555555555555555555555555555555 999900000000001111111111222222222233333333334444444444555555555566666666667777 678901234567890123456789012345678901234567890123456789012345678901234567890123 C1a TTAAAAATTTTATATTTAATTGATGAAATTGTAAATCCTTTTTTTTCAATTAAATCAATTGGTCATCAATGATATTGA C2c TTAAAAATTTTATATTTAATTGATGAAATTGTAAATCCTTTTTTTTCAATTAAATCAATTGGTCATCAATGATATTGA C2d TTAAAAATTTTATATTTAATTGATGAAATTGTAAATCCTTTTTTTTCAATTAAATCAATTGGTCATCAATGATATTGA C2e TTAAAAATTTTATATTTAATTGATGAAATTGTAAATCCTTTTTTTTCAATTAAATCAATTGGTCATCAATGATATTGA C2i TTAAAAATTTTATATTTAATTGATGAAATTGTAAATCCTTTTTTTTCAATTAAATCAATTGGTCATCAATGATATTGA C20 TTAAAAATTTTATATTTAATTGATGAAATTGTAAATCCTTTTTTTTCAATTAAATCAATTGGTCATCAATGATATTGA C2p TTAAAAATTTTATATTTAATTGATGAAATTGTAAATCCTTTTTTTTCAATTAAATCAATTGGTCATCAATGATATTGA

Fig. 2. Alignment of the sequences of the mitochondrial intergenic region found in the Serbian honey bees. The mtDNA fragment corresponds to positions 3363-3935 bp published by Crozier and Crozier (1993).

the hybrid population of $A$. $m$. carnica and A. m. macedonica from Albania (Tab. 3).

The data set included 12 haplotypes, seven from this work and five $(\mathrm{C} 2 \mathrm{a}, \mathrm{C} 2 \mathrm{~b}$, $\mathrm{C} 2 \mathrm{f}, \mathrm{C} 2 \mathrm{~g}$ and $\mathrm{C} 2 \mathrm{~h}$ ) which were previously published (Franck et al., 2000; Özdil et al., 2009) and included for comparison. In total, 573 base pairs (562 invariable sites, 8 variable sites and 3 gaps) were analyzed to identify haplotype relationships within the $\mathrm{C}$ evolutionary lineage. 
Table 2 .

Polymorphic sites (position in nucleotide changes and $\mathrm{bp}$ ) of the intergenic tRNAleu-cox2 region of $A$. mellifera $\mathrm{C}$-lineage. The mtDNA fragment corresponds to the positions 3363-3935 bp published by Crozier and Crozier (1993)

\begin{tabular}{||c|c|c|c|c|c|c|c|c||}
\hline \hline & $\begin{array}{c}\text { Genbank acces- } \\
\text { sion number }\end{array}$ & $\begin{array}{c}69 \\
(3432 \mathrm{bp})\end{array}$ & $\begin{array}{c}87 \\
(3450 \mathrm{bp})\end{array}$ & $\begin{array}{c}112 \\
(3475 \mathrm{bp})\end{array}$ & $\begin{array}{c}152 \\
(3515 \mathrm{bp})\end{array}$ & $\begin{array}{c}225 \\
(3488 \mathrm{bp})\end{array}$ & $\begin{array}{c}270 \\
(3633 \mathrm{bp})\end{array}$ & $\begin{array}{c}405 \\
(3768 \mathrm{bp})\end{array}$ \\
\hline C1a & JQ977699 & $\mathrm{C}$ & $\mathrm{T}$ & $\mathrm{T}$ & $\mathrm{T}$ & $\mathrm{G}$ & $\mathrm{T}$ & $\mathrm{C}$ \\
\hline C2c & JQ977700 & - & $\mathrm{T}$ & $\mathrm{T}$ & $\mathrm{T}$ & $\mathrm{G}$ & $\mathrm{T}$ & $\mathrm{C}$ \\
\hline C2d & JQ977701 & - & $\mathrm{T}$ & $\mathrm{T}$ & $\mathrm{T}$ & $\mathrm{G}$ & $\mathrm{C}$ & $\mathrm{T}$ \\
\hline C2e & JQ977702 & - & $\mathrm{T}$ & - & $\mathrm{T}$ & $\mathrm{G}$ & $\mathrm{C}$ & $\mathrm{T}$ \\
\hline C2i & JQ977703 & - & $\mathrm{T}$ & $\mathrm{T}$ & $\mathrm{T}$ & $\mathrm{A}$ & $\mathrm{C}$ & $\mathrm{T}$ \\
\hline C2o & JQ977704 & - & $\mathrm{T}$ & - & $\mathrm{A}$ & $\mathrm{G}$ & $\mathrm{C}$ & $\mathrm{T}$ \\
\hline C2p & JQ977705 & - & $\mathrm{C}$ & - & $\mathrm{T}$ & $\mathrm{G}$ & $\mathrm{C}$ & $\mathrm{T}$ \\
\hline \hline
\end{tabular}

Table 3 .

Number of analyzed colonies (n), haplotype frequency

and diversity (D) in the honey bee colonies from Serbia and the reference populations

\begin{tabular}{|c|c|c|c|c|c|c|c|c|c|}
\hline Population & $\mathrm{n}$ & C1a & C2c & C2d & C2e & C2i & C20 & C2p & D \\
\hline Banat (Serbia) & 13 & & & 0.615 & 0.231 & 0.077 & 0.077 & & 0.556 \\
\hline Syenichko-Peshterski (Serbia) & 10 & & & 0.400 & 0.400 & & & 0.200 & 0.640 \\
\hline Timok (Serbia) & 6 & 0.167 & 0.167 & 0.500 & & 0.167 & & & 0.667 \\
\hline Southeast (Serbia) & 8 & & & 0.750 & 0.250 & & & & 0.375 \\
\hline A. m. ligustica (reference) & 5 & 1.000 & & & & & & & 0.000 \\
\hline A. m. carnica (reference) & 10 & 0.100 & 0.100 & 0.500 & 0.300 & & & & 0.640 \\
\hline Hybrid population (reference) & 4 & & & 0.500 & & 0.500 & & & 0.500 \\
\hline A. m. macedonica (reference) & 5 & & & 1.000 & & & & & 0.000 \\
\hline
\end{tabular}

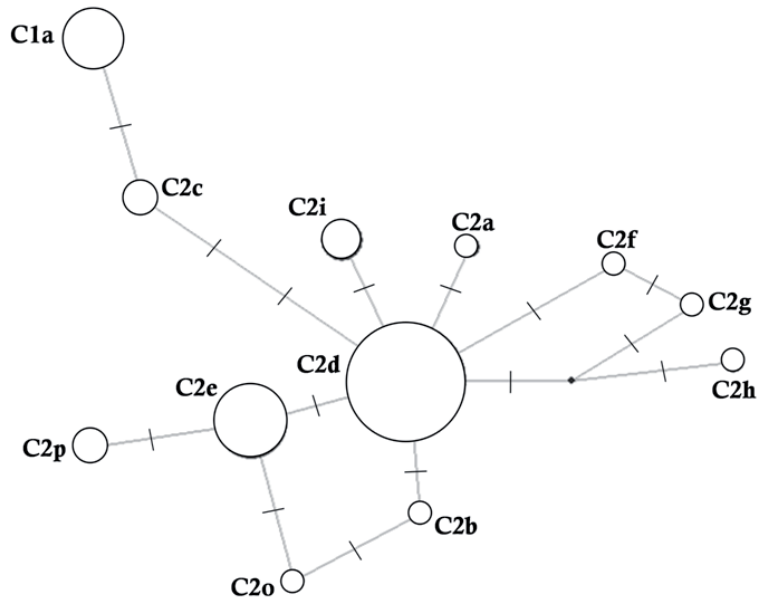

Fig. 3. Median-joining network connecting 12 haplotypes based on the sequence of the tRNA ${ }^{\text {leu }}$ cox2 intergenic region. Each circle represents a distinct haplotype. Tick marks along branches indicate the number of mutational changes between nodes. Black dots correspond to unsampled haplotypes. The size of the circles is proportional to the observed frequency of each haplotype. Haplotypes $\mathrm{C} 2 \mathrm{a}$ and $\mathrm{C} 2 \mathrm{~b}$ were described by Franck et al. (2000) while C2f, C2g and C2h by Özdil et al. (2009) in Turkey. 
Parsimony analyses resulted in a network with four unresolved connections (Fig. 3). The C2d haplotype occupied a central position in the network, whereas the most distant haplotype $\mathrm{Cla}$ which was present only in $A$. m. ligustica, showed five mutations with respect to C2d. Newly described haplotypes $\mathrm{C} 2 \mathrm{o}$ and $\mathrm{C} 2 \mathrm{p}$, were separated from $\mathrm{C} 2 \mathrm{e}$ by one single mutation each. One haplogroup was differentiated and separated, including three haplotypes (C2f, $\mathrm{C} 2 \mathrm{~g}$ and $\mathrm{C} 2 \mathrm{~h}$ ) present in A. m. meda and $A$. $m$. anatoliaca from Turkey.

\section{DISCUSSION}

The analysis of Serbian honey bees with molecular markers provides additional evidence of the underlying genetic diversity in the sequence of the intergenic tRNA $^{\text {leu-cox}} 2$ region. A total of seven honey bee mitochondrial haplotypes were detected in Serbia. Two newly described haplotypes, $\mathrm{C} 2 \mathrm{o}$ and $\mathrm{C} 2 \mathrm{p}$, defined after two newly detected polymorphic sites (one transversion and one transition), were found in the B and S regions, respectively. These findings indicate greater honey bee molecular diversity in Serbia compared to the other assessed Balkan countries, where present investigations and those of Muñoz et al. (2009) and Stevanovic et al. (2010) revealed only one (Republic of Macedonia), two (Albania) or four (Croatia) haplotypes. This is not surprising, as the territory of Serbia is a significant biodiversity center on the Balkan Peninsula. Serbia's status as a centre of biodiversity in Europe is to a high degree determined by its geological age, geomorphology, and climatic conditions and, in particular, by the role that the territory of Serbia played as an important refuge for a number of species during the Pleistocene glaciations (Hewitt et al., 1999). However, taking into consideration possible human-mediated queen importation, the high diversity of honey bees in Serbia could also be interpreted as the result of anthropogenic influences.

Honeybee colonies from the four Serbian regions present different haplotype frequency, $\mathrm{C} 2 \mathrm{~d}$ being more frequent in the southeast. Among the seven haplotypes found in Serbian honey bees, only C2d haplotype was detected in colonies from each analysed region, with frequencies of $0.750,0.615,0.500$ and 0.400 in bees from the $\mathrm{SE}, \mathrm{B}, \mathrm{T}$ and $\mathrm{S}$ regions, respectively. These results may be interpreted in many ways. Due to high frequency and widespread distribution in all Serbian regions, the $\mathrm{C} 2 \mathrm{~d}$ is perhaps the ancestral haplotype in Serbia. However, the C2d haplotype was previously found in Greece, with a frequency of 0.8 (Muñoz et al., 2009), and also in Albania, Bosnia and Herzegovina and the Republic of Macedonia (Stevanovic et al., 2010; present study). These findings may suggest gene flow and the natural northward migration of A. $m$. macedonica along the river basins of Vardar, Struma, Pcinja, south and great Morava up to Sava. The same could be inferred from the findings of $\mathrm{C} 2 \mathrm{i}$ in Serbian honey bees (with the frequencies of 0.167 and 0.077 in $\mathrm{T}$ and $\mathrm{B}$ regions, respectively), as this haplotype was detected only in A. m. macedonica from Albania (present study) and Greece (Muñoz et al., 2009). Beside the natural northward invasion of bees, introgression from $A$. m. macedonica into the Serbian populations could be due to human migrations that took place after the Ottoman invasions and after the First World War.

The haplotype $\mathrm{C} 2 \mathrm{e}$ was detected in honey bee populations from the S, SE and $B$ regions, with frequencies of $0.400,0.250$ and 0.231 , respectively. Such a detection could be interpreted as the consequence of the introduction of $A$. m. carnica honey bees from Croatia to Serbia most probably through human-mediated queen trade. This haplotype was first described in Croatian coastal honey bees with a high overall frequency (0.45) (Muñoz et al., 2009).

Other haplotypes detected in Serbian honey bees (C1a, C2c and $\mathrm{C} 2 \mathrm{i}$ ) possibly came later than $\mathrm{C} 2 \mathrm{~d}$ and $\mathrm{C} 2 \mathrm{e}$ as they are less frequent and their geographical distribution is not so widespread. C1a, C2c found only in bees from $\mathrm{T}$ region and $\mathrm{C} 2 \mathrm{i}$ 
found in $\mathrm{T}$ and $\mathrm{B}$ regions have also been detected in Italy, Slovenia and Greece, respectively. Such findings confirm the close relationship among the subspecies forming the $\mathrm{C}$ evolutionary group, as well as the intense commercial exchanges between beekeepers of this area.

The finding of $\mathrm{Cla}$ haplotype in honey bees from the Timok region suggests that introgression from A. m. ligustica might have occurred in these colonies. This is not surprising since $A$. $m$. ligustica is certainly the honey bee subspecies that is most implicated in human introductions because of its favorable characteristics (Franck et al., 2000). Two new haplotypes were observed solely in colonies from two regions: $\mathrm{C} 2 \mathrm{o}$ in $\mathrm{B}$ and $\mathrm{C} 2 \mathrm{p}$ in $\mathrm{S}$, although at different frequencies, 0.077 the first and 0.200 the second. These two haplotypes could be putative discriminant markers for the ecotypes dispersed in these regions, which should be contrasted with a wider sampling.

In conclusion, the honeybee colonies located in Serbia present a hybrid situation between A. m. carnica and $A$. $m$. macedonica, although introgression from A. m. ligustica has been also observed. On the other hand, two of the cytogenetic-described honeybee ecotypes (B and S) showed particular haplotypes, whose presence and frequency should be determined in posterior studies. The results found in this region confirm a preliminary analysis and should be considered in future conservation strategies and for pathogenparasites-tolerant breeding programs (Stevanovic et al., 2011).

\section{ACKNOWLEDGEMENT}

This study was supported
by the Ministry of Education
and Science of the Republic of Serbia
Grant No. III 46002 . Irene Muñoz is
supported by the Spanish Ministry of
Education.

\section{REFERENCES}

Bandelt H. J., Forster P., Rōhl A. (1999) - Median-joining networks for inferring intraspecific phylogenies. Mol. Biol. Evol., 16: 37-48.

Bouga M., Harizanis P. B., Kilias G., Alahiotis S. (2005) - Genetic divergence and phylogenetic relationships of honey bee Apis mellifera (Hymenoptera: Apidae) populations from Greece and Cyprus using PCR - RFLP analysis of three mtDNA segments. Apidologie, 36: 335-344.

Cirkovic D. (2002)-Reproductive-productive and hygienic-grooming characterization of Syenichko-Peshterski honey bee ecotype, MSc thesis, Belgrade University.

Crozier R. H., Crozier Y. H. (1993) - The mitochondrial genome of the honey bee Apis mellifera: complete sequence and genome organisation. Genetics, 133: 97-117.

Dedej S., Delaplane K. S., Gocaj E. (2000) - A technical and economic evaluation of beekeeping in Albania. Bee World, 81: 87-97.

Engel M. S. (1999) - The taxonomy of recent and fossil honey bees (Hymenoptera: Apidae, Apis). J. Hymenop. Res., 8: 165-196.

Franck P., Garnery L., Celebrano G., Solignac M., Cornuet J. M. (2000) - Hybrid origins of honey bees from Italy (Apis mellifera ligustica) and Sicily (A. m. sicula). Mol. Ecol., 9: 907-921.

Garnery L., Vautrin D., Cornuet J. M., Solignac M. (1991) - Phylogenetic relationships in the genus Apis inferred from mitochondrial DNA sequence data. Apidologie, 22: 87-92.

Hewitt G. M. (1999) - Post-glacial recolonization of European biota. Biol. J. Linn. Soc., 68: 87-112.

Ivanova E., Petrov P., Bouga M., Emmanouel N., Ivgin-Tukka R., Kence M. (2010) - Genetic variation in honey bee (Apis mellifera L.) populations from Bulgaria. J. Apic. Sci., 54: 49-59. 
KaToh K., Toh H. (2008) - Recent developments in the MAFFT multiple sequence alignment program. Briefings in Bioinformatics, 9: 286-298.

Martimianakis S., Klossa-Kilia E., Bouga M., Kilias G. (2011) - Phylogenetic relationships of Greek Apis mellifera subspecies based on sequencing of mtDNA segments (COI and ND5). J. Apic. Res., 50: 42-50.

Moritz R. F. A., Härtel S., Neumann P. (2005) - Global invasions of the western honey bee (Apis mellifera) and the consequences for biodiversity. Ecoscience, 12: 289-301.

Muñoz I., Dall'Olio R., Lodesani M., De la Rúa P. (2009) - Population genetic structure of coastal Croatian honeybees (Apis mellifera carnica). Apidologie, 40: 617-626.

Özdil F., Yildiz M. A., Hall H. G. (2009) - Molecular characterization of Turkish honeybee populations (Apis mellifera L.) inferred from mitochondrial DNA RFLP and sequence results. Apidologie, 40: 570-576.

Pejovic D. (2001) - Investigations of hygienic and grooming behaviour of honey bee subspecies Apis mellifera carnica in disease resistance, MSc thesis, Belgrade University.

Ruttner F. (1988) - Biogeography and Taxonomy of Honeybees, Springer-Verlag; Berlin, Germany, 284 pp.

Sheppard W. S., Meixner M. D. (2003) Apis mellifera pomonella, a new honey bee subspecies from Central Asia. Apidologie, 34: 367-375.

Sinacori A., Rinderer T. E., Lancaster V., Sheppard W. S. (1998) A morphological assessment of Apis mellifera from Palermo, Italy. Apidologie, 29: 481-490.

Stanimirovic Z., Popeskovic D., Pejovic D. (1999a) - Biodiversity of the honeybee Apis mellifera, Linne (1758), from the Yugoslav regions I - the biometric variability of the Banate and Sjenicko -Peshtersky ecotypes chromosomes. Acta Vet. Beograd, 49: 199-206.
Stanimirovic Z., Vucinic M., Stevanovic J. (1999b) - Biodiversity of the honeybee Apis mellifera, Linne (1758), from some Yugoslav regions: II - Ultrastructural chromosomal differences between Banat and Syenichko-Peshterski honeybee ecotype. Acta Vet. Beograd, 49: 207-214.

$\begin{array}{cccc}\text { Stanimirovic } & \text { Z., } & \text { Pejovic } & \text { D., } \\ \text { Stevanovic } & \text { J., } & \text { Vucinic } & \text { M., }\end{array}$
Mirilovic M. (2002) - Investigations of hygienic behaviour and disease resistance in organic beekeeping of two honeybee ecogeographic varieties from Serbia. Acta Vet. Beograd, 52: 169-180.

Stanimirovic Z., Stevanovic J., Andjelkovic M. (2005a) - Chromosomal diversity in Apis mellifera carnica from Serbia. Apidologie, 36: 31-42.

Stanimirovic Z., Stevanovic J., Cirkovic D. (2005b) - Behavioural defenses of the honey bee ecotype from Sjenica - Pester against Varroa destructor. Acta Vet. Beograd, 55: 69-82.

Stevanovic J. (2002) - Investigations of morphometric and chromosomal variability in diversity preserving of Carniolan honey bee (Apis mellifera carnica Pollmann, 1879) in Serbia, MSc thesis, Belgrade University.

Stevanovic J. (2007) - Ecological-ethological defence mechanisms of Apis mellifera carnica against ectoparasite Varroa destructor on the territory of Serbia. $\mathrm{PhD}$ dissertation, $\mathrm{PhD}$ thesis, Belgrade University.

Stevanovic J., Stanimirovic Z., Radakovic M., Kovacevic R. S. (2010) - Biogeographic study of the honey bee (Apis mellifera L.) from Serbia, Bosnia and Herzegovina and Republic of Macedonia based on mitochondrial DNA analyses. Rus. $J$. Genet., 46: 603-609.

Stevanovic J., Stanimirovic Z., Genersch E., Kovacevic S. R., Ljubenkovic J., Radakovic M., Aleksic N. (2011) - Dominance of Nosema ceranae in honey bees in the Balkan countries in the absence of symptoms of colony collapse disorder. Apidologie, 42: 49-58. 
Sušnik S., Kozmus P., Poklukar J., Whitfield C. W., Behura S. K., Meglič V. (2004)-Molecularcharacterisation Berlocher S. H., Clark A. G., of indigenous Apis mellifera carnica in Johnston J. S., Sheppard W. S., Slovenia. Apidologie, 35: 623-636.

Walsh P. S., Metzqer D. A., Higuchi R. (1991) - Chelex 100 as a medium for simple extraction of DNA for PCR-based typing from forensic material. Biotechniques, 10: 506-512. Smith D .R., Suarez A. V., Weaver D., Tsutsui N. D. (2006) - Thrice out of Africa: Ancient and recent expansions of the honey bee, Apis mellifera. Science, 314: 642-645.

\title{
RÓŻNORODNOŚĆ GENETYCZNA APIS MELLIFERA W SERBII NA PODSTAWIE BADAŃ MITOCHONDRIALNYCH
}

\author{
Muñoz I., Stevanovic J., \\ Stanimirovic Z., De la Rúa $P$.
}

St r e s z c z e n i e

Na terenie Serbii występują dwa podgatunki pszczoły miodenj: Apis mellifera carnica i A. m. macedonica. Oba te podgatunki należą do wschodnio-śródziemnomorskiej linii ewolucyjnej (C). Na podstawie analiz morfometrycznych oraz cytogenetycznych zidentyfikowano trzy ekotypy pszczoły miodnej występujące w określonych rejonach Serbii. W przedstawionych badaniach, do określenia różnorodności molekularnej serbskiej populacji pszczół wykorzystano wyniki analiz mitochondrialnych. Stwierdzono siedem haplotypów linii ewolucyjnej C, z których dwa to nieznane wcześniej haplotypy C2o i C2p o występowaniu ograniczonym do dwóch regionów, co w efekcie zwiększa liczbę haplotypów tej linii. Porównania z okolicznymi populacjami pszczół sugerują mieszanie się pszczół A. m. carnica i A. m. macedonica oraz introgresję A. m. ligustica. Uzyskane wyniki należy uwzględniać podczas ustalania programów hodowli zachowawczych oraz w programach hodowlanych zorientowanych na selekcję pszczół o zwiększonej tolerancji na patogeny i pasożyty.

Słowa kluczowe: Apis mellifera carnica, Apis mellifera macedonica, DNA mitochondrialne, region międzygenowy, haplotypy, sekwencjonowanie, genetyka populacyjna. 